А.В. Одокиенко

Наџиональный аэрокосмический университет имени Н.Е. Жуковского «ХАИ», Харьков

\title{
ОБОСНОВАНИЕ ОПТИМАЛЬНОСТИ ОПЕРАЦИИ ДЕЛЕНИЯ ОЦЕНОК ШУМОВЫХ ТЕМПЕРАТУР НА СМЕЖНЫХ ИНТЕРВАЛАХ НАБЛЮДЕНИЯ В РАДИОМЕТРЕ С НЕСТАБИЛЬНЫМ КОЭФФИЦИЕНТОМ УСИЛЕНИЯ
}

В статье обоснована оптимальность операции деления оценок иумовых температур на смежных интервалах наблюдения в радиометре с нестабильным коэффициентом усиления. Задача раздельной оптимизации статистических оченок иумовых температур случайных прочессов, наблюдаемых на двух смежных интервалах времени равной длительности при открытом и закрытом входе модуляционного радиометра с нестабильным коэффициентом усиления, решена методом максимального правдоподобия. Показано, что одной из существенных операщий синтезированного алгоритма первичной обработки сигналов является операция деления оценок их средних мощностей, измеренных на соседних временньх интервалах. Выведены аналитические выражения для предельных погрешностей оценок шумовой температуры исследуемого источника и нестабильности коэффициента усиления. Эти погрешности зависят от времени наблюдения, ширины полосы рабочих частот по входу, отношения сигнал/шум и нестабильности коэффициента усиления. Полученный алгоритм обработки сигналов целесообразно реализовывать в радиометрах, в состав додетекторных трактов которых входят недорогие низкостабильные усилители.

Ключевые слова: модуляционный радиометр, коэффиџиент усиления, метод максимального правдоподобия, обработка сигналов, оценка шумовой температуры.

\section{Введение}

Приемники собственного теплового излучения объектов в радиодиапазоне волн - радиометры применяют для решения задач радиофизики в интересах как научных исследований, так и различных отраслей народного хозяйства. С помощью радиометров измеряют шумовые температуры объектов, которые содержат информацию об их свойствах и свойствах среды распространения сигналов. Значительные трудности при оценивании шумовых температур исследуемых источников излучения связаны с устранением нестабильности усилителей, размещенных во входном тракте радиометра. Природа таких нестабильностей разнообразна, но наиболее значительный вклад вносят флуктуации температуры радиоэлементов, вызванные изменением как внешних условий проведения эксперимента, так и нагреванием в процессе работы. Поэтому разработчики современных радиометров, работа которых предполагает минимальное вмешательство инженеpa, обеспечивают стабильность температуры. Например, при размещении радиометров на искусственных спутниках земли [1-3] такая температурная стабилизация обеспечивается выбором солнечносинхронной орбиты, в других условиях - реализуют громоздкие системы термостабилизации [4] или сложные алгоритмы нулевого метода измерений [5].

Прежде всего, нестабильность усиления влияет на точностные характеристики измерений. С целью снижения этого влияния на измерения различными авторами предлагались разнообразные алгоритмы обработки сигналов, среди которых особо следует выделить использующие модуляцию полезного сигнала. Одним из первых удачных технических решений радиометров с модуляцией был радиометр Dicke [6]. В современной литературе ему часто ошибочно приписывают свойство полной компенсации нестабильности усиления. Но в силу мультипликативного воздействия нестабильности коэффициента усиления на интенсивность шумовых процессов полностью устранить его влияние в таком приемнике не удается.

В [7-10] синтезирован и исследован алгоритм обработки сигналов в модуляционном радиометре, предполагающий деление энергий сигналов, измеренных на соседних полуинтервалах функции модуляции (модуляция входных шумовых сигналов выполняется периодическим закрыванием и открыванием входа приемника). В настоящее время выполнены экспериментальные исследования, подтверждающие достоверность полученного алгоритма. В то же время интерес представляет решение оптимизационной задачи синтеза алгоритма в несколько иной постановке. Как показано в статье, новые результаты позволяют дополнительно вычленить особенности алгоритма с делением энергий и доказать принципиальную необходимость вторичной обработки - усреднения оценок радоияркостной температуры объекта. При синтезе алгоритма использованы элементы теории синтеза радиометрических устройств, систем и комплексов, изложенные в $[11,12]$.

Постановка задачи. Необходимо синтезировать и исследовать алгоритм оптимальной обработки стохастических сигналов радиотеплового излучения в приёмнике с флуктуациями усиления во входном тракте. 


\section{Изложение материалов исследования}

Обоснование структуры входного (додетекторного) тракта радиометрического приемника. При оценке статистических характеристик стохастического сигнала при нестабильном усилении минимальное количество оцениваемых неизвестных равняется трем. Один из них - оценка дисперсии (мощности, эффективной температуры) внутреннего шума. Эту оценку предположим известной. Оценить её можно с заданной степенью точности на этапе калибровки приемника непосредственно перед измерениями. Два оставшихся - оценка параметра сигнала (дисперсии, мощности, эффективной температуры) и оценка функции, описывающей нестабильность усиления, - могут быть оценены только в процессе измерений. Соответственно этому необходимы как минимум два независимых уравнения наблюдения. С этой целью целесообразно сформировать два математических (один физический) тракта, содержащих информацию о полезном сигнале и о флуктуации усиления. Это достигается использованием поочередного подключения и отключения приёмной антенны от входа приёмника. T.e. реализации основного принципа работы модуляционных радиометров с модуляцией сигнала функцией в виде меандра. Математические модели и физическое устройство, реализующее два независимых уравнения наблюдения, показаны на рис. 1.
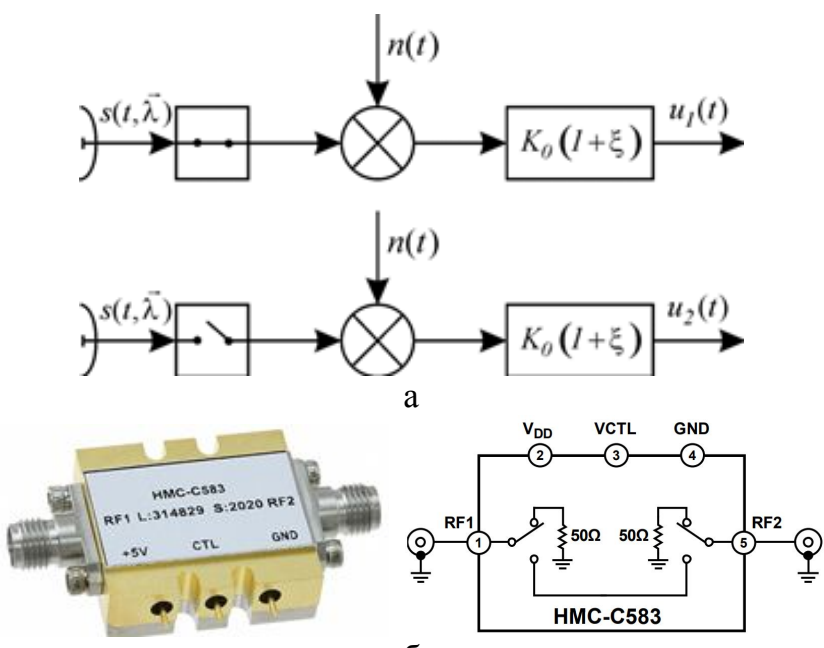

6

Рис. 1. Схема, соответствующая математическим моделям уравнений наблюдения (а) и переключатель HMC-C583 Analog Devices Inc. для диапазона частот 0,1-40 ГГц с его принципиальной схемой (б)

Уравнения наблюдения. Составим уравнения наблюдения соответственно приведенным рассуждениям и рис. 1, a). Учтём также, что измерения производятся в смежные короткие интервалы времени $\mathrm{t} \in\left(\mathrm{T}_{1}, \mathrm{~T}_{2}\right)$ и $\mathrm{t} \in\left(\mathrm{T}_{2}, \mathrm{~T}_{3}\right), \mathrm{T}_{2}-\mathrm{T}_{1}=\mathrm{T}_{3}-\mathrm{T}_{2}=\Delta \mathrm{T} / 2$, в течение которых изменяющийся во времени нестабильный коэффициент усиления $\mathrm{K}_{0}(1+\xi(\mathrm{t}))$ можно считать постоянным, т.е. $\mathrm{K}_{0}(1+\xi(\mathrm{t})) \approx \mathrm{K}_{0}(1+\xi)$.
В один из этих интервалов времени наблюдаются процесс, состоящий из суммы полезного шумового сигнала $s(t, \vec{\lambda})$ и помехи в виде внутреннего шума приемника $\mathrm{n}(\mathrm{t})$. В записи полезного сигнала $\mathrm{s}(\mathrm{t}, \vec{\lambda})$ учтена его зависимость от вектора параметров $\vec{\lambda}$, которыми могут быть как статистические характеристики стохастического сигнала (мощность или эффективная температура), так и электрофизические параметры объекта исследования. В другой интервал времени наблюдается только внутренний шум приемника. Простейшая идеализированная модель наблюдаемых процессов может быть представлена системой уравнений

$$
\begin{gathered}
\mathrm{u}_{1}(\mathrm{t})=[\mathrm{s}(\mathrm{t}, \vec{\lambda})+\mathrm{n}(\mathrm{t})] \mathrm{K}_{0}(1+\xi), \quad \mathrm{t} \in\left(\mathrm{T}_{1}, \mathrm{~T}_{2}\right), \\
\mathrm{u}_{2}(\mathrm{t})=\mathrm{n}(\mathrm{t}) \mathrm{K}_{0}(1+\xi), \quad \mathrm{t} \in\left(\mathrm{T}_{2}, \mathrm{~T}_{3}\right) .
\end{gathered}
$$

Процессы $\mathrm{s}(\mathrm{t}, \vec{\lambda})$ и $\mathrm{n}(\mathrm{t})$ на выходе линейной части приемника с коэффициентом усиления $\mathrm{K}_{0}$ сверхширокополосные, неразделимые и в идеализированном варианте их анализа и обработки полагаются гауссовыми процессами типа белого шума с нулевыми математическими ожиданиями $\langle\mathrm{s}(\mathrm{t})\rangle=0$, $\langle\mathrm{n}(\mathrm{t})\rangle=0$ и автокорреляционными и взаимно корреляционной функциями следующего вида:

$$
\begin{aligned}
\mathrm{R}_{\mathrm{s}}\left(\mathrm{t}_{1}, \mathrm{t}_{2}, \vec{\lambda}\right)=\left\langle\mathrm{s}\left(\mathrm{t}_{1}, \vec{\lambda}\right) \mathrm{s}\left(\mathrm{t}_{2}, \vec{\lambda}\right)\right\rangle=\frac{\mathrm{N}_{0 \mathrm{~s}}(\vec{\lambda})}{2} \delta\left(\mathrm{t}_{1}-\mathrm{t}_{2}\right) \\
\mathrm{R}_{\mathrm{n}}\left(\mathrm{t}_{1}, \mathrm{t}_{2}\right)=\left\langle\mathrm{n}\left(\mathrm{t}_{1}\right) \mathrm{n}\left(\mathrm{t}_{2}\right)\right\rangle=\frac{\mathrm{N}_{0 \mathrm{n}}}{2} \delta\left(\mathrm{t}_{1}-\mathrm{t}_{2}\right) \\
\left\langle\mathrm{s}\left(\mathrm{t}_{1}, \vec{\lambda}\right) \mathrm{n}\left(\mathrm{t}_{2}\right)\right\rangle=0
\end{aligned}
$$

где $\mathrm{N}_{0 \mathrm{~s}}(\vec{\lambda}) / 2, \mathrm{~N}_{0 \mathrm{n}} / 2$ - спектральные плотности мощности (СПМ) полезного случайного процесса и помехи, $\delta\left(\mathrm{t}_{1}-\mathrm{t}_{2}\right)$ - дельта-функция, $\langle\cdot\rangle-$ скобки статистического усреднения. СПМ являются двухсторонними, т.е. определенными на $f \in(-\infty, \infty)$.

Выше уже отмечалось, что нестабильность коэффициента усиления представлена функцией

$$
\mathrm{K}_{0} \xi(\mathrm{t})=\Delta \mathrm{K}(\mathrm{t})
$$

и изменяется во времени. Но на коротких интервалах наблюдения эта нестабильность полагается постоянной величиной

$$
\mathrm{K}_{0} \xi=\Delta \mathrm{K}=\text { const . }
$$

СПМ полезного сигнала и помехи связаны с эффективной шумовой (радиояркостной) температурой источника излучения $\mathrm{T}_{\mathrm{s}}$ и с эффективной шумовой температурой помехи $\mathrm{T}_{\mathrm{n}}$ таким образом:

$$
\mathrm{N}_{0 \mathrm{~s}}(\vec{\lambda})=\mathrm{k}_{\mathrm{B}} \mathrm{T}_{\mathrm{s}}(\vec{\lambda}), \mathrm{N}_{0 \mathrm{n}}=\mathrm{k}_{\mathrm{B}} \mathrm{T}_{\mathrm{n}},
$$

где $\mathrm{k}_{\mathrm{B}}$ - постоянная Больцмана.

СПМ исследуемого источника излучения $\mathrm{N}_{0 \mathrm{~s}}(\vec{\lambda}) / 2$ и температура $\mathrm{T}_{\mathrm{s}}(\vec{\lambda})$ зависят от его электрофизических и геометрических параметров $\vec{\lambda}$. В данном подразделе решается оптимизационная задача оценок СПМ $\mathrm{N}_{0 \mathrm{~s}}$ или соответствующей ей температуры $\mathrm{T}_{\mathrm{S}}$ совместно с оценкой коэффициента неста- 
бильности $\xi=$ const. СПМ внутреннего шума $\mathrm{N}_{0 \text { n }}$ или соответствующая ей температура внутреннего шума $T_{n}$, оцениваемые на этапе калибровки радиометра, полагается известной. В некоторых случаях общем виде математическое описание этой задачи рассматривается относительно всех параметров $\vec{\lambda}$, входящих в систему уравнений наблюдения (1), (2), в число которых добавим и параметр нестабильности $\xi$.

Оптимальные оценки параметров $\vec{\lambda}$ и $\xi$ уравнений наблюдения. Так как оцениваемые параметры являются постоянными величинами и их вероятностные распределения неизвестны, то решение данной оптимизационной задачи целесообразно искать в рамках метода максимального правдоподобия. Рассмотрим эту задачу в постановке раздельной оптимизации оценок искомых параметров в моменты времени $\mathrm{t} \in\left(\mathrm{T}_{1}, \mathrm{~T}_{2}\right), \mathrm{t} \in\left(\mathrm{T}_{2}, \mathrm{~T}_{3}\right)$. В таком варианте раздельной оптимизации оценки параметров находим в результате отдельного поиска максимумов двух функций правдоподобия для случайных процессов, наблюдаемых в соответствующие моменты времени. Для гауссовых уравнений наблюдения (1) и (2) с нулевым средним функции правдоподобия представим следующими выражениями:

$$
\begin{aligned}
& \forall \mathrm{t} \in\left(\mathrm{T}_{1}, \mathrm{~T}_{2}\right) \quad \mathrm{p}\left[\mathrm{u}_{1}(\mathrm{t}) \mid \vec{\lambda}, \xi\right]=\mathrm{k}_{1}(\vec{\lambda}, \xi) \times \\
& \times \exp \left[-\frac{1}{2} \int_{\mathrm{T}_{1}}^{\mathrm{T}_{2}} \int_{\mathrm{T}_{1}}^{\mathrm{T}_{2}} \mathrm{u}_{1}\left(\mathrm{t}_{1}\right) \mathrm{W}_{\mathrm{u}_{1}}\left(\mathrm{t}_{1}, \mathrm{t}_{2}, \vec{\lambda}, \xi\right) \mathrm{u}_{1}\left(\mathrm{t}_{2}\right) \mathrm{dt}_{1} \mathrm{dt}_{2}\right] \text {, } \\
& \forall \mathrm{t} \in\left(\mathrm{T}_{2}, \mathrm{~T}_{3}\right) \quad \mathrm{p}\left[\mathrm{u}_{2}(\mathrm{t}) \mid \xi\right]=\mathrm{k}_{2}(\xi) \times \\
& \times \exp \left[-\frac{1}{2} \int_{\mathrm{T}_{2} \mathrm{~T}_{2}}^{\mathrm{T}_{3}} \int_{\mathrm{T}_{3}}^{\mathrm{T}_{3}} \mathrm{u}_{2}\left(\mathrm{t}_{1}\right) \mathrm{W}_{\mathrm{u}_{2}}\left(\mathrm{t}_{1}, \mathrm{t}_{2}, \xi\right) \mathrm{u}_{2}\left(\mathrm{t}_{2}\right) \mathrm{dt}_{1} \mathrm{dt}_{2}\right] \text {, }
\end{aligned}
$$

где $\mathrm{W}_{\mathrm{u}_{1}\left(\mathrm{u}_{2}\right)}\left(\mathrm{t}_{1}, \mathrm{t}_{2}, \vec{\lambda}, \xi\right)$ - функции, обратные корреляционным функциям $\mathrm{R}_{\mathrm{u}_{1}\left(\mathrm{u}_{2}\right)}\left(\mathrm{t}_{1}, \mathrm{t}_{2}, \vec{\lambda}, \xi\right)$, определяются из интегральных уравнений обращения

$\int_{\mathrm{T}_{2}}^{\mathrm{T}_{1}} \mathrm{R}_{\mathrm{u}_{1}}\left(\mathrm{t}_{1}, \mathrm{t}_{2}, \vec{\lambda}, \xi\right) \mathrm{W}_{\mathrm{u}_{1}}\left(\mathrm{t}_{2}, \mathrm{t}_{3}, \vec{\lambda}, \xi\right) \mathrm{dt}_{2}=\delta\left(\mathrm{t}_{1}-\mathrm{t}_{3}\right)$,

$$
\int_{\mathrm{T}_{2}}^{\mathrm{T}_{3}} \mathrm{R}_{\mathrm{u}_{2}}\left(\mathrm{t}_{1}, \mathrm{t}_{2}, \xi\right) \mathrm{W}_{\mathrm{u}_{2}}\left(\mathrm{t}_{2}, \mathrm{t}_{3}, \xi\right) \mathrm{dt}_{2}=\delta\left(\mathrm{t}_{1}-\mathrm{t}_{3}\right),
$$

$\mathrm{k}_{1}(\vec{\lambda}, \xi), \mathrm{k}_{2}(\xi)$ - коэффициенты, зависяп от $\vec{\lambda}$ и $\xi$.

Корреляционные функции каждого из наблюдений найдены в следующем виде:

$$
\begin{gathered}
\mathrm{R}_{\mathrm{u}_{1}}\left(\mathrm{t}_{1}, \mathrm{t}_{2}, \vec{\lambda}, \xi\right)=\left\langle\mathrm{u}_{1}\left(\mathrm{t}_{1}\right) \mathrm{u}_{1}\left(\mathrm{t}_{2}\right)\right\rangle= \\
=(1+\xi)^{2} \cdot\left[\mathrm{R}_{\mathrm{s}}\left(\mathrm{t}_{1}, \mathrm{t}_{2}, \vec{\lambda}\right)+\mathrm{R}_{\mathrm{n}}\left(\mathrm{t}_{1}, \mathrm{t}_{2}\right)\right]= \\
=\mathrm{K}_{0}^{2}(1+\xi)^{2} \frac{\mathrm{N}_{0 \mathrm{~s}}+\mathrm{N}_{0 \mathrm{n}}}{2} \delta\left(\mathrm{t}_{1}-\mathrm{t}_{2}\right), \\
\mathrm{R}_{\mathrm{u}_{2}}\left(\mathrm{t}_{1}, \mathrm{t}_{2}, \vec{\lambda}, \xi\right)=\left\langle\mathrm{u}_{2}\left(\mathrm{t}_{1}\right) \mathrm{u}_{2}\left(\mathrm{t}_{2}\right)\right\rangle=(1+\xi)^{2} \times \\
\times \mathrm{R}_{\mathrm{n}}\left(\mathrm{t}_{1}, \mathrm{t}_{2}\right)=\mathrm{K}_{0}^{2}(1+\xi)^{2} \cdot\left(\mathrm{N}_{0 \mathrm{n}} / 2\right) \cdot \delta\left(\mathrm{t}_{1}-\mathrm{t}_{2}\right) .
\end{gathered}
$$

Оптимальные оценки параметров $(\vec{\lambda}, \xi)$ находим из условия максимумов функций правдоподобия (9), (10) или, учитывая монотонность экспонент, из эквивалентных условий

$$
\begin{gathered}
\frac{\partial \ln \mathrm{p}\left[\mathrm{u}_{1}(\mathrm{t}) \mid \vec{\lambda}, \xi\right]}{\partial \lambda_{\mu}}=0, \\
\frac{\partial \ln \mathrm{p}\left[\mathrm{u}_{2}(\mathrm{t}) \mid \xi\right]}{\partial \xi}=0 .
\end{gathered}
$$

Дифференцируя (15), (16) получим:

$$
\begin{aligned}
& -\frac{1}{2} \int_{\mathrm{T}_{1}}^{\mathrm{T}_{2}} \int_{\mathrm{T}_{1}}^{\mathrm{T}_{2}}\left[\frac{\partial}{\partial \lambda_{\mu}} \mathrm{R}_{\mathrm{u}_{1}}\left(\mathrm{t}_{1}, \mathrm{t}_{2}, \hat{\vec{\lambda}}, \hat{\xi}\right)\right] \mathrm{W}_{\mathrm{u}_{1}}\left(\mathrm{t}_{1}, \mathrm{t}_{2}, \hat{\vec{\lambda}}, \hat{\xi}\right) \mathrm{dt}_{1} \mathrm{dt}_{2}- \\
& -\frac{1}{2} \int_{\mathrm{T}_{1}}^{\mathrm{T}_{2} \mathrm{~T}_{2}}\left[\frac{\partial}{\partial \lambda_{\mu}} \mathrm{W}_{\mathrm{u}_{1}}\left(\mathrm{t}_{1}, \mathrm{t}_{2}, \hat{\vec{\lambda}}, \hat{\xi}\right)\right] \mathrm{u}_{1}\left(\mathrm{t}_{1}\right) \mathrm{u}_{1}\left(\mathrm{t}_{2}\right) \mathrm{dt}_{1} \mathrm{dt}_{2}=0, \\
& -\frac{1}{2} \int_{\mathrm{T}_{2}}^{\mathrm{T}_{3}} \int_{\mathrm{T}_{2}}^{\mathrm{T}_{3}}\left[\frac{\partial}{\partial \xi} \mathrm{R}_{\mathrm{u}_{2}}\left(\mathrm{t}_{1}, \mathrm{t}_{2}, \hat{\xi}\right)\right] \mathrm{W}_{\mathrm{u}_{2}}\left(\mathrm{t}_{1}, \mathrm{t}_{2}, \hat{\xi}\right) \mathrm{dt}_{1} \mathrm{dt}_{2}- \\
& -\frac{1}{2} \int_{\mathrm{T}_{2}}^{\mathrm{T}_{3} \mathrm{~T}_{2}}\left[\frac{\partial}{\partial \xi} \mathrm{W}_{\mathrm{u}_{2}}\left(\mathrm{t}_{1}, \mathrm{t}_{2}, \hat{\xi}\right)\right] \mathrm{u}_{2}\left(\mathrm{t}_{1}\right) \mathrm{u}_{2}\left(\mathrm{t}_{2}\right) \mathrm{dt}_{1} \mathrm{dt}_{2}=0,
\end{aligned}
$$

где " $\wedge "$ - знак оценки, $\lambda_{\mu}$ может быть параметром $\xi$ и условно считаться одним из параметров $\vec{\lambda}$.

Для решения уравнений (17), (18) необходимо найти функции, обратные корреляционным функциям. Подставим выражение (14) в уравнение (12), а (15) в (13). В результате найдем функции, обратные корреляционным функциям в следующем виде:

$$
\begin{gathered}
\mathrm{W}_{\mathrm{u}_{1}}\left(\mathrm{t}_{1}, \mathrm{t}_{3}, \vec{\lambda}, \xi\right)=\delta\left(\mathrm{t}_{1}-\mathrm{t}_{3}\right) \times \\
\times 2 /\left(\mathrm{K}_{0}^{2}(1+\xi)^{2}\left[\mathrm{~N}_{0 \mathrm{~s}}(\vec{\lambda})+\mathrm{N}_{0 \mathrm{n}}\right]\right), \\
\mathrm{W}_{\mathrm{u}_{2}}\left(\mathrm{t}_{1}, \mathrm{t}_{3}, \xi\right)=\frac{2}{\mathrm{~K}_{0}^{2}(1+\xi)^{2} \mathrm{~N}_{0 \mathrm{n}}} \delta\left(\mathrm{t}_{1}-\mathrm{t}_{3}\right) .
\end{gathered}
$$

Подставив (13) и (19) в уравнение правдоподобия (17), находим алгоритм обработки сигналов для оценки СПМ сигнала и шума

$$
\mathrm{N}_{0 \mathrm{~s}}(\hat{\vec{\lambda}})+\mathrm{N}_{0 \mathrm{n}}=\frac{2}{\mathrm{~K}_{0}^{2}(1+\hat{\xi})^{2} \delta(0)(\Delta \mathrm{T} / 2)} \int_{\mathrm{T}_{1}}^{\mathrm{T}_{2}} \mathrm{u}_{1}^{2}(\mathrm{t}) \mathrm{dt} .
$$

Используя одну из моделей дельта-функции $\delta\left(\mathrm{t}_{1}-\mathrm{t}_{2}\right)=\int_{-\infty}^{\infty} \mathrm{e}^{\mathrm{j} 2 \pi \mathrm{f}\left(\mathrm{t}_{1}-\mathrm{t}_{2}\right)} \mathrm{df}=\lim _{\Delta \mathrm{F} \rightarrow \infty} \int_{-\Delta \mathrm{F}}^{\Delta \mathrm{F}} \mathrm{e}^{\mathrm{j} 2 \pi \mathrm{f}\left(\mathrm{t}_{1}-\mathrm{t}_{2}\right)} \mathrm{df}$, при $\mathrm{t}_{1}=\mathrm{t}_{2}$ получим

$$
\delta(0)=\left.\delta\left(t_{1}-t_{2}\right)\right|_{t_{1}=t_{2}}=\lim _{\Delta F \rightarrow \infty} \int_{-\Delta F}^{\Delta F} d f=\lim _{\Delta F \rightarrow \infty}(2 \Delta F)=\infty,
$$

где $2 \Delta \mathrm{F}$ - ширина спектра случайного процесса. Для белых шумов она равна бесконечности, т.е.

$$
\mathrm{N}_{0 \mathrm{~s}}(\hat{\vec{\lambda}})=\lim _{\Delta \mathrm{F} \rightarrow \infty} \frac{1}{(\Delta \mathrm{T} / 2) \Delta \mathrm{FK}_{0}^{2}(1+\hat{\xi})^{2}} \int_{0}^{\mathrm{T}} \mathrm{u}^{2}(\mathrm{t}) \mathrm{dt}-\mathrm{N}_{0 \mathrm{n}} .
$$


Для небелых шумов, т.е. случайных процессов с ограниченной полосой $\Delta \mathrm{F}$

$$
\mathrm{N}_{0 \mathrm{~s}}(\hat{\vec{\lambda}})=1 /\left((\Delta \mathrm{T} / 2) \Delta \mathrm{FK}_{0}^{2}(1+\hat{\xi})^{2}\right) \cdot \int_{0}^{\mathrm{T}} \mathrm{u}^{2}(\mathrm{t}) \mathrm{dt}-\mathrm{N}_{0 \mathrm{n}} .
$$

Решая аналогичным образом уравнение (18), находим оптимальную оценку множителя интенсивности $(1+\hat{\xi})^{2}$

$$
(1+\hat{\xi})^{2}=\frac{2}{\mathrm{~K}_{0}^{2}(\Delta \mathrm{T} / 2) \delta(0) \mathrm{N}_{0 \mathrm{n}}} \int_{\mathrm{T}_{1}}^{\mathrm{T}_{2}} \mathrm{u}_{2}^{2}(\mathrm{t}) \mathrm{dt} .
$$

Подставляя (25) в (21) находим

$$
\begin{gathered}
\mathrm{N}_{0 \mathrm{~s}}(\hat{\vec{\lambda}})+\mathrm{N}_{0 \mathrm{n}}=\left(\mathrm{P}_{\mathrm{cp} 1} / \mathrm{P}_{\mathrm{cp} 2}\right) \cdot \mathrm{N}_{0 \mathrm{n}}, \\
\mathrm{P}_{\mathrm{cp} 1,2}=\frac{1}{(\Delta \mathrm{T} / 2)} \int_{\Delta \mathrm{T} / 2} \mathrm{u}_{1,2}^{2}(\mathrm{t}) \mathrm{dt}-
\end{gathered}
$$

средняя мощность наблюдения.

Из (26) получаем оценку $\mathrm{N}_{0 \mathrm{~s}}(\hat{\vec{\lambda}})$, в ней исключено влияние множителя нестабильности $(1+\xi)^{2}$

$$
\mathrm{N}_{0 \mathrm{~s}}(\hat{\vec{\lambda}})=\left(\mathrm{P}_{\mathrm{cpl}} / \mathrm{P}_{\mathrm{cp} 2}\right) \cdot \mathrm{N}_{0 \mathrm{n}}-\mathrm{N}_{0 \mathrm{n}}=\mathrm{N}_{0 \mathrm{n}}\left(\mathrm{P}_{\mathrm{cp} 1} / \mathrm{P}_{\mathrm{cp} 2}-1\right) \text {. }
$$

Переходя к шумовым температурам согласно (8), оценим эффективную температуру:

$$
\mathrm{T}_{\mathrm{s}}(\hat{\vec{\lambda}})=\mathrm{T}_{\mathrm{n}}(\vec{\lambda})\left(\mathrm{P}_{\mathrm{cp} 1} / \mathrm{P}_{\mathrm{cp} 2}-1\right) .
$$

Таким образом, в результате решения оптимизационной задачи оценивания СПМ $\mathrm{N}_{0 \mathrm{~s}}(\hat{\vec{\lambda}})$, а также связанных с ней параметров $\hat{\vec{\lambda}}$ и $\mathrm{T}_{\mathrm{s}}(\hat{\vec{\lambda}})-$ шумовой температуры установлено, что операция деления оценок средних мощностей шумовых сигналов, наблюдаемых в смежных периодах времени равной длительности, составляет основу оптимального алгоритма их обработки. Следует отметить, что ввиду малой длительности интервалов $\Delta \mathrm{T} / 2$, на которых оцениваются средние мощности (27), алгоритмы (28), (29) следует считать первичными, а полученные оценки дополнительно усреднять.

Предельные погрешности оценок эффективной шумовой температуры и множителя нестабильности. В соответствии с теорией Рао-Крамера предельная погрешность оценки искомого параметра случайного процесса равна величине обратной среднему значению второй производной от логарифма функционала правдоподобия, найденному при истинном или оценочном значении этого параметра

$$
\begin{gathered}
\sigma_{\lambda}^{2}=\left\{-\left.\left\langle\frac{\partial^{2} \ln \mathrm{P}[\mathrm{u}(\mathrm{t}) \mid \lambda]}{\partial^{2} \lambda}\right\rangle\right|_{\vec{\lambda}_{\vec{\lambda}} \approx \hat{\mathrm{\lambda}}}\right\}^{-1}= \\
=\left\{-\frac{1}{2} \iint_{\mathrm{T} T}\left[\frac{\partial}{\partial \lambda} \mathrm{R}\left(\mathrm{t}_{1}, \mathrm{t}_{2}, \lambda\right)\right]\left[\frac{\partial}{\partial \lambda} \mathrm{W}\left(\mathrm{t}_{1}, \mathrm{t}_{2}, \lambda\right)\right] \mathrm{dt}_{1} \mathrm{dt}_{2}\right\}^{-1},
\end{gathered}
$$

где $\mathrm{T}$ - интервал наблюдения случайного процесса $u(t)$.

При наблюдении процесса $\mathrm{u}_{1}(\mathrm{t})$ на интервале $\mathrm{t} \in\left(\mathrm{T}_{1}, \mathrm{~T}_{2}\right), \quad \mathrm{T}_{2}-\mathrm{T}_{1}=\Delta \mathrm{T} / 2$ для функционала прав- доподобия (9) $\mathrm{P}\left[\mathrm{u}_{1}(\mathrm{t}) \mid \lambda\right]$ и оптимального алгоритма (23) оценки $\mathrm{N}_{0 \mathrm{~s}}$

$$
\begin{gathered}
\sigma_{\mathrm{N}_{0 \mathrm{~s}}}^{2}=\left\{-\frac{1}{2} \int_{\mathrm{T}_{1}}^{\mathrm{T}_{2}} \int_{1}^{\mathrm{T}_{2}}\left[\frac{\partial}{\partial \mathrm{N}_{0 \mathrm{~s}}} \mathrm{R}_{\mathrm{u}_{1}}\left(\mathrm{t}_{1}, \mathrm{t}_{2}, \lambda\right)\right] \times\right. \\
\left.\times\left[\frac{\partial}{\partial \mathrm{N}_{0 \mathrm{~s}}} \mathrm{~W}_{\mathrm{u}_{1}}\left(\mathrm{t}_{1}, \mathrm{t}_{2}, \lambda\right)\right] \mathrm{dt}_{1} \mathrm{dt}_{2}\right\}^{-1}=\frac{2\left(\widehat{\mathrm{N}}_{0 \mathrm{~s}}+\mathrm{N}_{0 \mathrm{n}}\right)^{2}}{(\Delta \mathrm{T} / 2) \delta(0)} .
\end{gathered}
$$

Предельная флуктуационная чувствительность радиометра на интервале наблюдения $(\Delta \mathrm{T} / 2)$ равна

$$
\sigma_{\mathrm{N}_{0 \mathrm{~s}}}=\sqrt{\frac{2\left(\widehat{\mathrm{N}}_{0 \mathrm{~s}}+\mathrm{N}_{0 \mathrm{n}}\right)^{2}}{(\Delta \mathrm{T} / 2) \delta(0)}}=\frac{\sqrt{2}\left(\widehat{\mathrm{N}}_{0 \mathrm{~s}}+\mathrm{N}_{0 \mathrm{n}}\right)}{\sqrt{(\Delta \mathrm{T} / 2) \lim _{\Delta \mathrm{F} \rightarrow \infty} 2 \Delta \mathrm{F}}} .
$$

Для процессов с конечной полосой

$$
\sigma_{\mathrm{N}_{0 \mathrm{~s}}}=\sqrt{2}\left(\widehat{\mathrm{N}}_{0 \mathrm{~s}}+\mathrm{N}_{0 \mathrm{n}}\right) / \sqrt{\Delta \mathrm{T} \Delta \mathrm{F}} .
$$

При выводе этой формулы полагалось, что СПМ $\mathrm{N}_{0 \mathrm{n}}$, определяемая в процессе калибровки радиометра является известной. Множитель нестабильности $(1+\xi)^{2}$ определяется в результате решения второй оптимизационной задачи (18) на соседнем интервале наблюдения $\mathrm{t} \in\left(\mathrm{T}_{2}, \mathrm{~T}_{3}\right), \mathrm{T}_{3}-\mathrm{T}_{2}=\Delta \mathrm{T} / 2 \quad$ и также полагается известным. Поэтому, как и коэффициент усиления $\mathrm{K}_{0}$, так и множитель $(1+\xi)^{2}$ здесь отсутствуют. При наблюдении процесса $\mathrm{u}_{2}(\mathrm{t})$ на интервале $\mathrm{t} \in\left(\mathrm{T}_{2}, \mathrm{~T}_{3}\right)$ для функционала правдоподобия (10) $\mathrm{P}\left[\mathrm{u}_{2}(\mathrm{t}) \mid \xi\right]$ и оптимального алгоритма (25) дисперсия оценки $\xi$

$$
\begin{gathered}
\sigma_{\xi}^{2}=\left\{-\frac{1}{2} \int_{\mathrm{T}_{2}}^{\mathrm{T}_{3}} \int_{\mathrm{T}_{2}}^{\mathrm{T}_{3}}\left[\frac{\partial}{\partial \xi} \mathrm{R}_{\mathrm{u}_{2}}\left(\mathrm{t}_{1}, \mathrm{t}_{2}, \lambda\right)\right] \times\right. \\
\left.\times\left[\frac{\partial}{\partial \xi} \mathrm{W}_{\mathrm{u}_{2}}\left(\mathrm{t}_{1}, \mathrm{t}_{2}, \lambda\right)\right] \mathrm{dt}_{1} \mathrm{dt}_{2}\right\}^{-1}=(1+\hat{\xi})^{2} /(2(\Delta \mathrm{T} / 2) \delta(0)),
\end{gathered}
$$

а потенциальная флуктуационная чувствительность к нестабильности

$$
\sigma_{\xi}=\frac{1+\hat{\xi}}{\sqrt{2(\Delta \mathrm{T} / 2) \delta(0)}}=(1+\hat{\xi}) / \sqrt{2 \Delta \mathrm{T} \lim _{\Delta \mathrm{F} \rightarrow \infty} \Delta \mathrm{F}} .
$$

Для процессов с конечной рабочей полосой частот соответственно получим

$$
\sigma_{\xi}^{2}=(1+\hat{\xi})^{2} /(2 \Delta \mathrm{T} \Delta \mathrm{F}), \quad \sigma_{\xi}=(1+\hat{\xi}) / \sqrt{2 \Delta \mathrm{T} \Delta \mathrm{F}},
$$

т.е. был получен алгоритм, нивелирующий нестабильность коэффициента усиления разной природы происхождения в додетекторной части радиометра.

\section{Выводы}

Решена задача раздельной оптимизации оценок СПМ (эффективной шумовой температуры) собственного излучения исследуемого объекта и множителя нестабильности коэффициента усиления усилителя 
во входном (додетекторном) тракте приемника. Доказано, что основой синтезированного алгоритма первичной обработки шумовых процессов является операция деления оценок средних мощностей наблюдаемых процессов на смежных интервалах времени равной длительности. Определены предельные погрешности оценок шумовой температуры исследуемого источника и нестабильности коэффициента усиления.

\section{Список литературы}

1. Alimenti F. A space-based $90 \mathrm{GHz}$ radiometer for the observation of solar flares: Feasibility study / F. Alimenti et al. // 2009 European Microwave Conference (EuMC). - Rome, 2009. - P. 982-985. doi: 10.23919/EUMC.2009.5296238.

2. Peng J. Soil Moisture Active/Passive L-Band Microwave Radiometer Postlaunch Calibration / J. Peng et al. // IEEE Transactions on Geoscience and Remote Sensing. - Sept. 2017. Vol. 55(9). - P. 5339-5354. doi: 10.1109/TGRS.2017.2705342.

3. Microwave Scanner-Sounder MTVZA-GY on New Russian Meteorological Satellite Meteor-M No. 2: Modeling, Calibration, and Measurements / L.M. Mitnik, V.P. Kuleshov, M.L. Mitnik, A.M. Streltsov, G.M. Chernyavsky and I.V. Cherny // IEEE Journal of Selected Topics in Applied Earth Observations and Remote Sensing. - July 2017. - Vol. 10(7). P. 3036-3045. doi: 10.1109/JSTARS.2017.2695224.

4. Microwave Radar and Radiometric Remote Sensing / F.T. Ulaby and D.G. Long // Michigan, University of Michigan Press. Ann Arbor, 2014. - P. 262-293.

5. Moon N.W. and Kim Y.H. Temperature Drift Compensation Using Multiple Linear Regression for a $W$-Band Total Power Radiometer / N.W. Moon and Y.H. Kim // IEEE Sensors Journal. 2015. - Vol. 15(8). - P. 4612-4620.

6. Филатов А.В. Новые принципы построения высокочувствительного микроволнового радиометра нулевого типа с многоканальным приемником / А.В. Филатов // Физические основы приборостроения, 2017. - №6, 2(24). - C. 67-75. doi: 10.25210/jfop-1702-067075.

7. Kemppainen $S$. Thermal stabilized front-end $P C B$ with active cold calibration load for L-band radiometer / S. Kemppainen et al. // 2007 IEEE International Geoscience and Remote Sensing Symposium. - Barcelona, 2017. P. 4433-4436. doi: 10.1109/IGARSS. 2007.4423838.

8. Dicke R.H. The measurement of thermal radiation at microwave frequencies / R.H. Dicke // Rev. Sci. Instrum., July 1946. - Vol. 17(7). - P. 268-275.

9. Pavlikov V.V. The New Type of Chopper Radiometer / V. V. Pavlikov, A. D. Sobkolov // Ultrawideband and Ultrashort Impulse Signals. (UWBUSIS 2016) : proc. $8^{\text {th }}$ Intern. conf., 5-11 Sept., 2016, Odesa, Ukraine. - Kharkov, 2016. - P. 205-208. doi: 10.1109/UWBUSIS.2016.7724189

10. Statistical Synthesis of Chopper Radiometric Systems / V.K. Volosyuk, V.F. Kravchenko, V.V. Pavlikov, V.I. Pustovoit // Doklady Physics. - 2013. - Vol. 58, № 1. - P. 29-32.

11. Pavlikov V.V. Radiometer with signal energies ratio / V.V. Pavlikov, S.S. Zhyla, A.V. Odokienko, M.O. Antonov // IEEE Radar Methods and Systems Workshop. (RMSW 2016) : proc. Intern. conf., 27-28 Sept., 2016, Kyiv, Ukraine. - Kyiv. P. 99-102. doi: 10.1109/RMSW.2016.7778560

12. Development of Digital Part of Ratio-Type Radiometer / V.V. Pavlikov, A.V. Odokiyenko, A.V. Sobkolov, K.N. Nezhalskaya, M.O. Antonov // The Experience of Designing and Application of CAD Systems in Microelectronics. CADSM'2017: proc. 14th Int. conf., 2017. - P. 122-124. doi: 10.1109/CADSM.2017.7916100.

\section{Надійшла до редколегї 18.12.2017}

Рецензент: д-р техн. наук, проф. Л.Ф. Купченко, Харківський національний університет Повітряних Сил імені Івана Кожедуба, Харків.

\section{ОБГРУНТУВАННЯ ОПТИМАЛЬНОСТІ ОПЕРАЦІЇ РОЗПОДІЛУ ОЦІНОК ШУМОВОЇ ТЕМПЕРАТУРИ НА СУМІЖНИХ ІНТЕРВАЛАХ СПОСТЕРЕЖЕННЯ У РАДІОМЕТРІ ІЗ НЕСТАБІЛЬНИМ КОЕФІЦІЄНТОМ ПОСИЛЕННЯ}

\section{О.В. Одокієнко}

У статті обтрунтована оптимальність операиії розподілу оцінок иумових температур на суміжних інтервалах спостереження у радіометрі з нестабільним коефічієнтом посилення. Завдання роздільної оптимізачії статистичних оцінок шумових температур випадкових прочесів, які спостерігаються на двох суміжснх інтервалах часу рівної тривалості при відкритому і закритому вході модулячійного радіометра із нестабільним коефіцієнтом посилення, вирішена методом максимальної правдоподібності. Показано, щзо однією з істотних операцій синтезованого алгоритму первинної обробки сигналів є операція розподілу оцінок їх середніх потужностей, виміряних на сусідніх часових інтервалах. Виведено аналітичні вирази для граничних похибок очінок шумової температури досліджуваного джерела $і$ нестабільності коефіцієнта посилення. Ці похибки залежать від часу спостереження, иирини смуги робочих частот за входом, відношення сигнал / шум і нестабільності коефіцієнта посилення. Отриманий алгоритм обробки сигналів доцільно реалізовувати в радіометрах, до складу додетекторних трактів яких входять недорогі низкостабільні підсилювачі.

Ключові слова: модулячійний радіометр, коефіцієнт посилення, метод максимальної правдоподібності, обробка сигналів, оцінка шумової температури.

\section{JUSTIFICATION FOR OPTIMIZATION OF OPERATION OF NOISE TEMPERATURE ESTIMATES DIVISION AT THE ADJACENT OBSERVATION INTERVALS IN A RADIOMETER WITH AN UNSTABLE AMPLIFICATION FACTOR}

\section{O.V. Odokienko}

In the article an optimization of operation of noise temperature estimates division at the adjacent observation intervals in a radiometer with an unstable amplification factor was justified. The separate optimization problem of statistical estimates of random processes noise temperatures observed on two contiguous time intervals of equal length with opened and closed input of an unstable amplification factor modulation radiometer was solved using the method of Maximum Likelihood Estimations. It was shown, that one of the significant operations of the synthesized primary signal processing algorithm is the division operation of the signals average powers estimates, changed on the adjacent time intervals. The analytical margin of errors expressions for noise temperature estimates of investigated source and amplification factor instability were derived. These margins of errors are dependent on observation time, width of input working frequencies, signal to noise ratio and amplification factor instability. The obtained signal processing algorithm is reasonable to use in radiometers, pre-detector paths of which contain rather cheap low-stable amplifiers.

Keywords: modulation radiometer, amplifying factor, noise temperature estimate, method of Maximum Likelihood Estimations, signal processing, noise temperature estimation. 